\title{
Self-induced Correction of the Genetic Defect in Tyrosinemia Type I
}

\author{
Eli Anne Kvittingen, * Helge Rootwelt, * Ruud Berger, ${ }^{*}$ and Per Brandtzaeg \\ ${ }^{*}$ Institute of Clinical Biochemistry, University of Oslo Rikshospitalet, N-0027 Oslo, Norway; ${ }^{\ddagger}$ Laboratory for Metabolic Diseases, \\ Wilhelmina Kinderzikenhuis, Utrecht, The Netherlands; and ${ }^{8}$ Institute of Pathology, University of Oslo, Rikshospitalet, \\ N-0027 Oslo, Norway
}

\begin{abstract}
A mosaic pattern of immunoreactive fumarylacetoacetase (FAH) protein was found in liver tissue in 15 of 18 tyrosinemia type I patients of various ethnic origins. One additional patient had variable levels of FAH enzyme activity in liver tissue. In four patients exhibiting mosaicism of FAH protein, analysis for the tyrosinemia-causing mutations was performed in immunonegative and immunopositive areas of liver tissue by restriction digestion analysis and direct DNA sequencing. In all four patients the immunonegative liver tissue contained the FAH mutations demonstrated in fibroblasts of the patients. In the immunopositive nodules of regenerating liver tissue one of the mutated alleles apparently had reverted to the normal genotype. This genetic correction was observed for three different tyrosinemia-causing mutations. In each case a mutant AT nucleotide pair was reverted to a normal GC pair. (J. Clin. Invest. 1994. 94:1657-1661.) Key words: liver disease $\cdot$ amino acid metabolism - mosaicism • mutagenesis $\cdot$ carcinogenesis
\end{abstract}

\section{Introduction}

Hereditary tyrosinemia type I (McKusick 27670) is caused by a severe deficiency of fumarylacetoacetase (FAH) ${ }^{1}$ (E.C. 3.7.1.2), the last enzyme in tyrosine degradation. The disorder is of autosomal recessive inheritance and is characterized by hypophosphatemic rickets due to renal tubular dysfunction and progressive liver disease with pronounced regeneration. It often leads to development of hepatocellular carcinoma if the patients survive for some years (1). The disorder occurs worldwide but with particular high frequency in Quebec, Canada.

Recently we reported a mosaic pattern of immunoreactive FAH protein in liver tissue of five Norwegian tyrosinemia patients, and in two of the patients some areas of liver tissue contained up to $50 \%$ residual FAH activity (2). We have now

Address correspondence to E.A. Kvittingen, Institute of Clinical Biochemistry, Rikshospitalet, N-0027 Oslo, Norway.

Received for publication 7 March 1994 and in revised form 30 June 1994.

1. Abbreviations used in this paper: APAAP, alkaline phosphatase/antialkaline phosphatase; FAH, fumarylacetoacetase.

J. Clin. Invest.

(c) The American Society for Clinical Investigation, Inc.

$0021-9738 / 94 / 10 / 1657 / 05 \$ 2.00$

Volume 94, October 1994, 1657-1661 extended this investigation to 19 tyrosinemia patients of various ethnic origins. We furthermore report the results of mutation analysis of liver tissue from areas with or without immunoreactive FAH protein in four patients exhibiting the FAH mosaicism in liver tissue. In these patients the "reappearance" of immunoreactive FAH protein appeared to be caused by a reversion of the primary point mutation to the normal nucleotide in one of the alleles.

\section{Methods}

Patients. 18 tyrosinemia patients of various ethnic origins were investigated by immunohistochemistry for a mosaic pattern of immunoreactive FAH protein in liver tissue (obtained at liver transplantation). In one additional patient, from whom only frozen liver tissue was available, FAH activity was determined in several small sections of the tissue. In three Norwegians and one Pakistani, patients with known mutations in the FAH gene and a mosaic pattern of FAH protein in liver tissue, immunopositive and immunonegative areas of liver tissue were subjected to mutation analysis. None of the four patients showed immunoreactive FAH protein in Western blots of fibroblast extracts. Patient 1 (patient 2 in reference 2), transplanted at six years of age without hepatocellular carcinoma, was compound heterozygous for a G to $A$ in position +5 in intron 12 (IVS12 $\mathrm{g}^{+5} \rightarrow \mathrm{a}$ ) and an unknown mutation (3). Patient 2, transplanted at age four without evidence of cancer, was compound heterozygous for the IVS12 $\mathrm{g}^{+5} \rightarrow$ a mutation and a $\mathrm{G}^{1069} \rightarrow \mathrm{T}$ nonsense mutation (E357X) (4). Patient 3, transplanted at age 10 due to hepatocellular carcinoma, was homozygous for $\mathrm{G}^{1009} \rightarrow \mathrm{A}$, a combined splice and missense mutation (G337S) (3), and patient 4 , transplanted at age 3 without cancer, was homozygous for $G^{192} \rightarrow T$, a splice site mutation (5).

Immunohistochemistry and mutation analysis in liver sections. Immunohistochemistry was performed on 5 - $\mu \mathrm{m}$ sections of formalin-fixed liver tissue with an alkaline phosphatase/anti-alkaline phosphatase (APAAP) method as described previously (2). For mutation analysis the sections were not counterstained with Hematoxylin and Eosin after immunohistochemical staining, as hematoxylin may inhibit PCR reactions. Liver tissue corresponding to immunopositive and immunonegative areas $(\sim 5 \times 5 \mathrm{~mm})$ as indicated in Fig. $1(\sim 2-2.5 \times$ the original size), was removed with a scalpel and transferred to a microcentrifuge tube. Buffer, 20-40 $\mu$ l, containing $0.05 \mathrm{M} \mathrm{KCl}, 0.01 \mathrm{M}$ Tris $/ \mathrm{HCl} \mathrm{pH}$ $8.3,2.5 \mathrm{mM} \mathrm{MgCl} 2,0.01 \%$ gelatine, $0.45 \% \mathrm{NP} 40,0.45 \%$ Tween, and $0.07 \mathrm{mg} / \mathrm{ml}$ proteinase $\mathrm{K}$ was added, and the tubes were incubated overnight at $50^{\circ} \mathrm{C}$. Details of the PCR reactions and the primers used for each of the mutations have been reported previously $(3,5)$. The IVS $12 \mathrm{~g}^{+5} \rightarrow$ a mutation (patient 1 and 2 ) is detected by amplification and digestion of a 109-bp product surrounding the mutation. One mismatch primer together with the mutation creates a restriction site for Asp700 digesting the mutated allele into 73- and 36-bp fragments. The second mutation in patient $2, \mathrm{G}^{1069} \rightarrow \mathrm{T}$ is detected by amplification of $108 \mathrm{bp}$ with two mismatches in one primer creating, with the normal allele, but not with the mutated allele, a restriction site for Asp700 digesting the normal allele to fragments of 79 and $29 \mathrm{bp}$. For the $\mathrm{G}^{1009} \rightarrow A$ mutation 

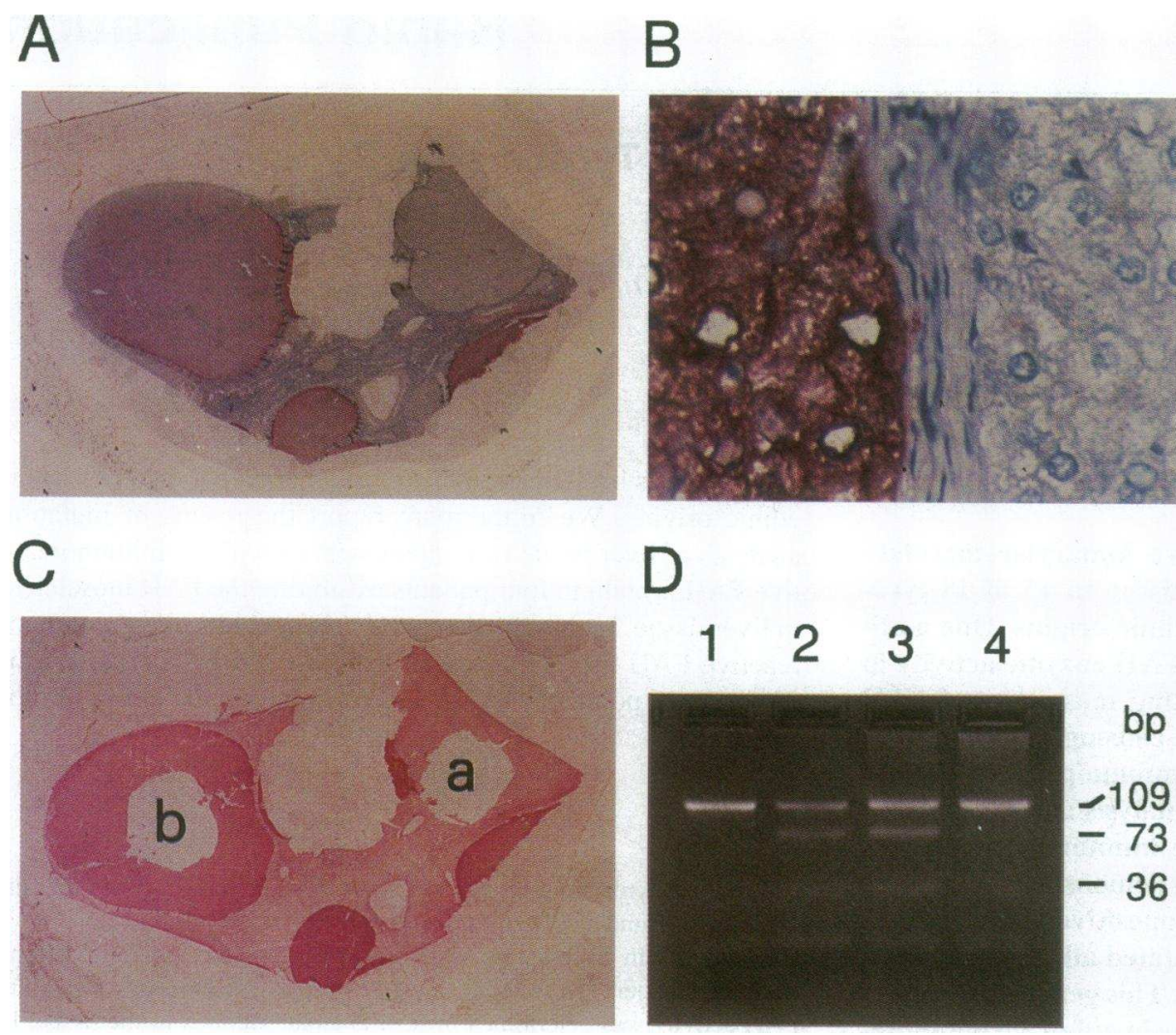

Figure 1. Immunohistochemistry of liver tissue and mutation analysis by restriction digestion of PCR products in patient 1 . (A) Overview of a section stained with APAAP and lightly counterstained with $\mathrm{H} \& \mathrm{E}$, shows one large immunopositive regenerative nodule (red) to the left and two positive areas at the lower edge. $(B)$ Higher magnification shows the clear-cut delineation between immunopositive and immunonegative cells. $(C)$ Serial section adjacent to that in $A$, stained with APAAP (no counterstain) shows sampling sites of the immunonegative (area $a$ ) and immunopositive (area $b$ ) tissue removed for mutation analysis. $(D)$ Agarose gel electrophoresis of Asp700 digested 109-bp PCR product surrounding the IVS12 $\mathrm{g}^{+5}$ $\rightarrow$ a mutation. The normal allele is undigested whereas the mutated allele is digested to fragments of 73 and $36 \mathrm{bp}$; lane I, control fibroblasts; lane 2, patient's fibroblasts; lane 3 , immunonegative liver tissue ( $C$, area $a$ ); lane 4 , immunopositive liver tissue $(C$, area $b)$.

(patient 3) a 298-bp segment was amplified. This mutation creates a restriction site for PvuII and, when the mutation is present, the 298-bp fragment is digested to fragments of 218 and $80 \mathrm{bp}$. The $\mathrm{G}^{192} \rightarrow \mathrm{T}$ mutation is detected by amplification of $61 \mathrm{bp}$, followed by NlaIII digestion of the mutated allele into fragments of 35 and $26 \mathrm{bp}$. The normal allele remains undigested. Direct automatic sequencing of the PCR products was performed as previously described (3).

\section{Results}

Immunohistochemical investigation of liver tissue from the 18 tyrosinemia patients, revealed a mosaic pattern in 9 Norwegian, 1 Syrian, 1 Australian, and 4 Pakistani patients. In one additional Norwegian patient assay of FAH activity in various section of liver tissue showed a range from $<2$ to $30 \%$ of normal activity, indicating mosaicism. In one of the three patients not showing mosaicism only one paraffin block of liver tissue was available for investigation, thus mosaicism may be present in other parts of the liver. In two patients 8-12 paraffin blocks were investigated without evidence of mosaicism. The parents of the four patients subjected to mutation analysis all have FAH activities in their fibroblasts compatible with a carrier state of tyrosinemia, and the mutations of the patients were verified in one or both of the respective parents.

Fig. 1 shows the immunohistochemical staining and the result of mutation analysis in patient 1 . Fig. $1 A$ (APAAP lightly counterstained with $\mathrm{H} \& \mathrm{E}$ ) demonstrates that some regeneration nodules are clearly immunopositive (red color), while other areas are immunonegative. At higher magnification (Fig. $1 B$ ) the clear-cut delineation between immunonegative and immunopositive cells is apparent. Fig. $1 C$ shows a section (adjacent to that in Fig. $1 A$ ) stained only with APAAP from which an immunonegative (area $a$ ) and an immunopositive (area $b$ ) tissue sample have been removed for mutation analysis.

Fig. $1 \mathrm{D}$ shows gel electrophoresis of the Asp700 digests of the 109-bp PCR product from a normal control (lane 1), fibroblast extract from the patient (lane 2), liver tissue from the immunonegative sample (Fig. $1 C$, area $a$ ) (lane 3 ) and liver tissue from the immunopositive sample (Fig. $1 C$, area $b$ ) (lane 4 ). In the latter, only a very small fraction of the PCR product is digested. Sequencing data of the PCR products from a normal control (Fig. $2 A$ ), the immunonegative liver tissue (Fig. $2 B$ ) and the immunopositive liver tissue (Fig. $2 C$ ) of patient 1 , support a substantial shift from the mutated $A$, for which the patient was heterozygous, to a normal $G$ nucleotide in the immunopositive liver tissue.

The results of patient 2, are given in Fig. 3. Liver tissue from an immunonegative (Fig. $3 \mathrm{~B}$, area $a$ ) and an immunopositive (area $b$ ) area was analyzed for the two mutations in this patient. Fig. $3 C$ shows restriction digestion by Asp700 of the PCR product surrounding the IVS $12 \mathrm{~g}^{+5} \rightarrow \mathrm{a}$ mutation from fibroblast extracts of a normal control (lane 1 ) and the patient (lane 2) and from the immunonegative liver tissue (Fig. $3 B$, area $a$ ) (lane 3 ) and immunopositive liver tissue (Fig. $3 B$, area b) (lane 4 ) of the patient. A major part of the PCR product from the immunopositive liver tissue is not digested, indicating absence of the mutation. Sequencing data supported the reversion of the mutation (data not shown). In cells from three additional immunopositive areas from this patient, the IVS 12 $\mathrm{g}^{+5} \rightarrow \mathrm{a}$ was reverted in all the regeneration nodules. The $\mathrm{G}^{1069} \rightarrow \mathrm{T}$ mutation was present in all the four immunopositive as well as the immunonegative areas investigated.

Immunohistochemical staining of liver tissue from patient 

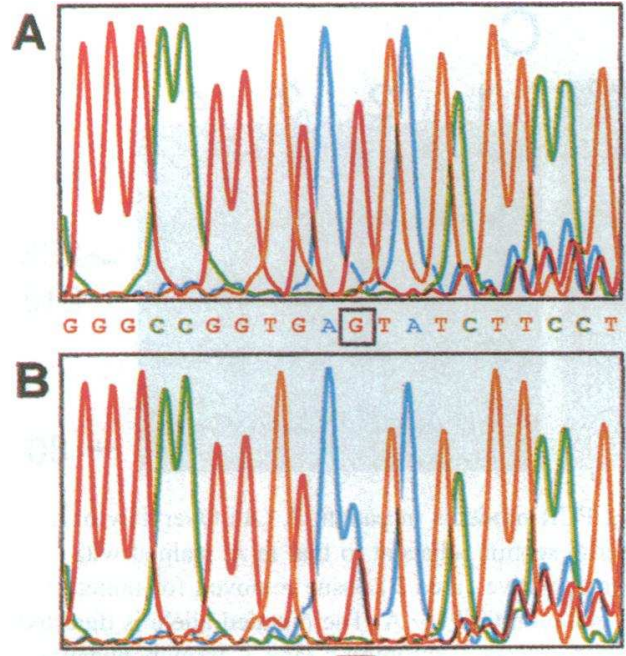

G G G C C G G T G A R T A T C T T C C T

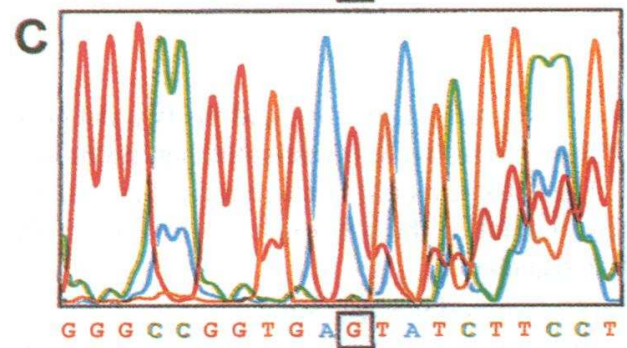

Figure 2. Direct sequencing raw data of the 109-bp PCR product surrounding the IVS12 $\mathrm{g}^{+5} \rightarrow \mathrm{a}$ mutation. (A) Control fibroblasts. (B) Immunonegative liver tissue (Fig. $1 C$, area $a$ ) from patient 1. (C) Immunopositive liver tissue (Fig. $1 C$, area $b$ ) from patient 1 . The squares indicate nucleotide position IVS12 $+5 . R$ indicates $\mathrm{G}$ and $\mathrm{A}$.

3, homozygous for $\mathrm{G}^{1009} \rightarrow \mathrm{A}$, is shown in Fig. $4, A$ and $B$. Restriction digestion by PvuII of the 298-bp product (Fig. $4 C$ ) showed no digestion of control fibroblasts (lane 1 ) and complete digestion of the product from the patient's fibroblasts (lane 2) and from the immunonegative liver tissue (Fig. $4 \mathrm{~B}$, area $a$ ) (lane 3 ). Both undigested and digested products from the immu- nopositive liver tissue (Fig. $4 \mathrm{~B}$, area $\mathrm{b}$ ) (lane 4 ), suggested absence of the mutation in one allele. Sequencing data of the 298-bp PCR product from control fibroblasts, the immunonegative liver tissue, and the immunopositive liver tissue of patient 3, are shown in Fig. 5, $A, B$, and $C$, respectively, confirming a heterozygous genotype for a mutant $A$ and the normal $G$ in the immunopositive liver tissue. No other sequence differences were found in the PCR products from the immunonegative and immunopositive liver tissue of the patient.

In patient 4 (Fig. 6), who is homozygous for the $G^{192} \rightarrow T$ mutation, restriction analysis (Fig. $6 C$ ) again indicates a reversion of the mutation in one allele in the immunopositive liver tissue (Fig. $6 B$, area $b$ ) (lane 4), in contrast to homozygosity for the mutation, with complete digestion, in the immunonegative tissue (Fig. $6 \mathrm{~B}$, area $a$ ) (lane 3 ) and in fibroblasts (lane 2) from the patient.

In all patients the results were reproduced in 3-6 liver sections of the respective areas.

\section{Discussion}

Some problems adhered to the mutation analysis of the formalinfixed liver sections. The obtainable length of the PCR product varied considerably from one paraffin block to another. Presumably, the tissue fixation or other factors may have degraded the DNA considerably and in some blocks only very short PCR products could be amplified. Furthermore, routine tissue preparation is performed without precautions for DNA contamination. Careful precautions in preparation of the tissue sections had to be observed to avoid interfering DNA contamination. In Fig. $4 C$ and $6 C$ (lanes 3 ) representing immunonegative liver tissue from the patients, trace amounts of undigested PCR product, not containing the mutation, are visible. This could be due to unavoidable contamination of normal DNA derived either from the positive area in the same liver section or, more likely, from other sources. In patients 1 and 2, the immunopositive tissue did not show a complete reversion of the mutation, as visualized by weak bands of 73 and 36 bp (Fig. $1 D$ and $3 C$, lanes 4 ). This could also be due to DNA contamination, but might as well be caused by (tissue) contribution of nuclei from non-hepatocyte cells which have retained the mutation.
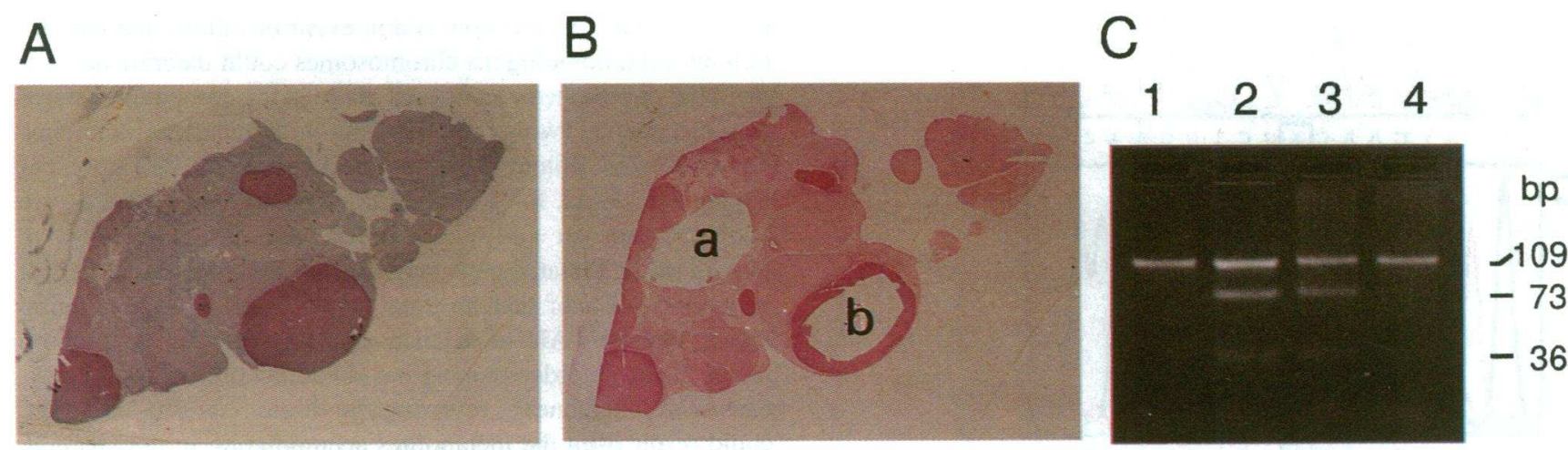

Figure 3. Immunohistochemistry of liver tissue and mutation analysis by restriction digestion of PCR products in patient 2. (A) Overview of a section, stained with APAAP and H\&E, shows several immunoreactive regeneration nodules. $(B)$ Serial section adjacent to that in $A$, stained with APAAP (no counterstain), shows the sampling sites of the immunonegative (area $a$ ) and immunopositive (area $b$ ) tissue removed for mutation analysis. $(C)$ Agarose gel electrophoresis of Asp 700 digested 109-bp PCR product surrounding the IVS12 $\mathrm{g}^{+5} \rightarrow$ a mutation. The normal allele is undigested, whereas the mutated allele is digested to 73 and $36 \mathrm{bp}$; lane 1 , control fibroblasts; lane 2, patient's fibroblasts; lane 3 , immunonegative liver tissue $(B$, area $a)$; lane 4 , immunopositive liver tissue $(B$, area $b)$. 

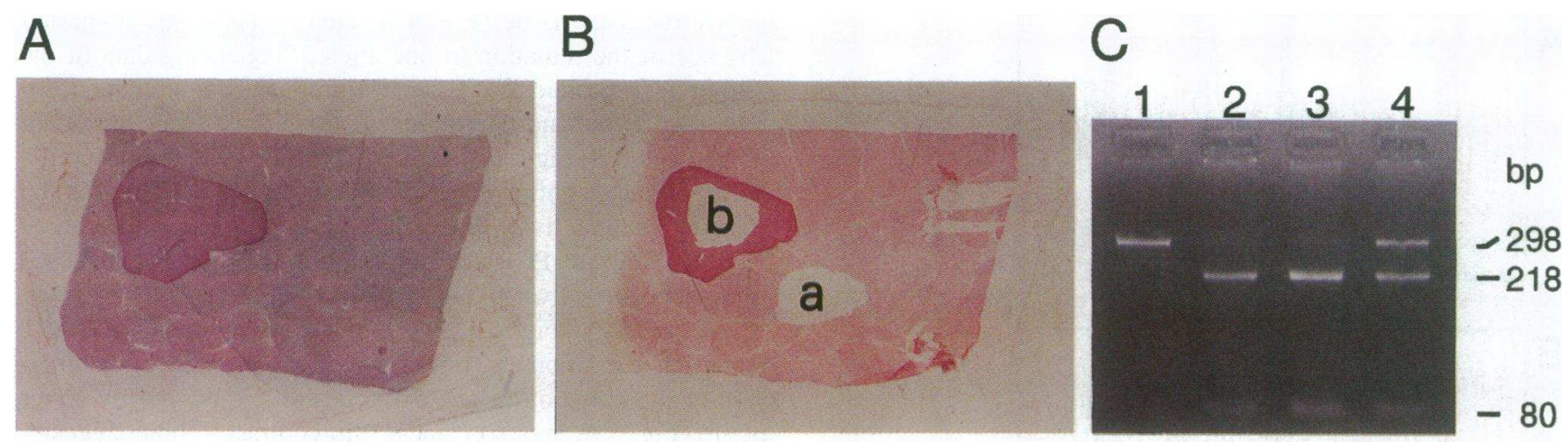

Figure 4. Immunohistochemistry of liver tissue and mutation analysis by restriction digestion of PCR products in patient 3. $(A)$ Overview of a liver section, stained with APAAP \& HE, shows one immunopositive nodule to the left. $(B)$ Serial section adjacent to that in $A$, stained with APAAP (no counterstain), shows the sampling sites of the immunonegative (area $a$ ) and immunopositive (area $b$ ) tissue removed for mutation analysis. C: Agarose gel electrophoresis of Pvull digested 298-bp PCR product surrounding the mutation $\mathrm{G}^{1009} \rightarrow$ A. The mutated allele is digested to 218 and $80 \mathrm{bp}$, whereas the normal allele remains undigested; lane 1, control fibroblasts; lane 2, patient's fibroblasts; lane 3, immunonegative liver tissue (Fig. $B$, area $a$ ); lane 4, immunopositive liver tissue (Fig. $B$, area $b$ ).

A mosaic pattern of FAH activity and immunoreactive protein appears to be common in liver tissue from tyrosinemia patients as 16 of 19 patients of various ethnic origins showed the phenomenon. The patients investigated represent a selected group since they were all liver transplanted and none of the patients were transplanted in early infancy. The youngest patient
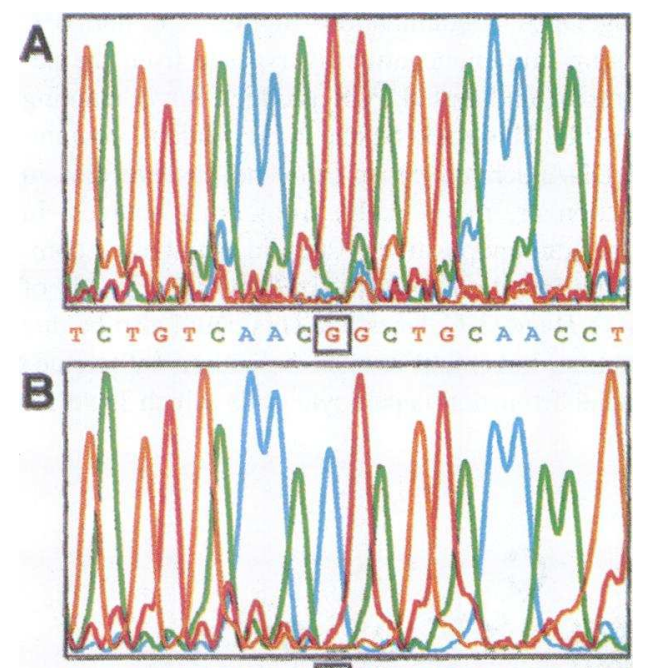

T C T G T C A A C A G C T G C A A C C T

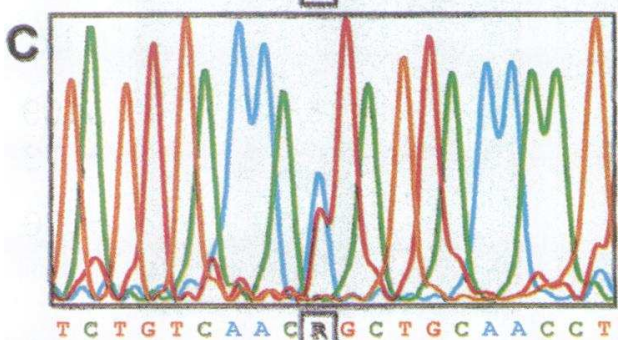

Figure 5. Direct sequencing raw data of the 298 -bp $P C R$ product surrounding the mutation $G^{1009} \rightarrow A$. ( $A$ ) Control fibroblasts. $(B)$ Immunonegative liver tissue (Fig. $4 B$, area $a$ ) from patient 3. (C) Immunopositive tissue (Fig. $4 B$, area $b$ ) from patient 3 . The squares indicate nucleotide position 1009. $R$ indicates $\mathrm{G}$ and $\mathrm{A}$. with mosaicism, however, was 11 months, and the two patients from whom 8-12 liver blocks were investigated and no mosaicism found, were 5 and 10 years old. An age related ascertainment bias may therefore not be the sole explanation of the high incidence of FAH mosaicism.

In the immunopositive nodules investigated in the four patients subjected to mutation analysis, the mosaicism apparently was caused by a reversion of the primary point mutation in one allele. Alternative explanations may, however, account for the phenomenon in non-investigated nodules of the four patients and in other patients exhibiting the phenomenon. A second site mutation somewhere in the exons inducing conformational changes stabilizing the transcript or protein sufficiently to give immunoreactivity, and possibly enzyme activity, may explain the mosaic pattern of FAH protein. Second-site mutation in the codon of the primary defect may also, in certain cases, establish a codon for the correct amino acid.

In the compound heterozygote patient the same mutation was reverted to wild type in all four nodules investigated. This could be accidental due to the low number of nodules investigated. On the other hand, mutation liability is sequence dependent $(6,7)$, thus one of the mutations may revert more easily than the other. A gene conversion event or mitotic recombination between homologous chromosomes could theoretically explain the appearance of a normal allele in the compound heterozygote patient. Two of the patients with reverted mutations, however, were homozygous for their mutations, and no pseudogenes for $\mathrm{FAH}$, for contribution of wild-type sequences, are known. Also, an early embryonic mutation with selective growth of the mutated cells may account for the mosaicism, but a high incidence of such an event would indicate a precipitating factor. As the FAH mosaicism appears frequent, a common factor of the disorder causing the phenomenon, is not unlikely. Chemical mutagenesis, reverting the disease-causing mutation, could result from the metabolites accumulating in tyrosinemia. Tyrosine degradation takes place mainly in the hepatocytes and when FAH is deficient these cells accumulate the highest amount of the alkylating metabolites fumaryl- (and maleyl-) acetoacetate. These metabolites may directly or indirectly cause the reversion, although direct mutagenic effect of the metabolites has yet to be demonstrated. Cancer development is, how- 

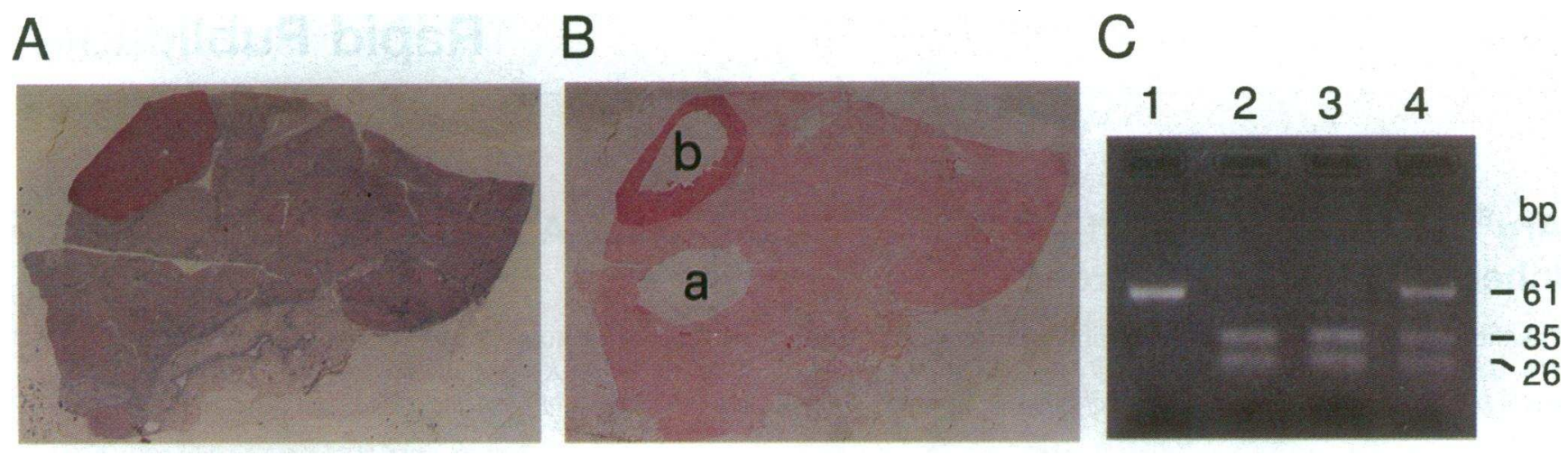

Figure 6. Immunohistochemistry of liver tissue and mutation analysis by restriction digestion of PCR products in patient 4. $(A)$ Overview of a liver section, stained with APAAP and H\&E, shows one immunopositive regeneration nodule at the upper edge. $(B)$ Serial section adjacent to that in $A$, stained with APAAP (no counterstain) shows the sampling sites of the immunonegative (area $a$ ) and immunopositive (area $b$ ) tissue removed for mutation analysis. $(C)$ Agarose gel electrophoresis of NlaIII-digested 61-bp PCR product surrounding the $\mathrm{G}^{192} \rightarrow \mathrm{T}$ mutation. The normal allele is undigested, whereas the mutated allele is digested to fragments of 35 and $26 \mathrm{bp}$; lane 1, control fibroblasts; lane 2, patient's fibroblasts; lane 3 , immunonegative liver tissue $(B$, area $a)$; lane 4 , immunopositive liver tissue $(B$, area $b)$.

ever, a major feature of tyrosinemia, and tyrosinemia livers removed at transplantation may show multifocal hepatocellular carcinomas (8). Even if the metabolites are not direct mutagens, the compounds are toxic and induce cell necrosis with a subsequent accelerated regeneration of hepatocytes. Rapidly replicating cells are generally prone to mutations. Stable mutations are continuously made during cell replication, but a selection pressure is needed for a mutated cell to proliferate and to be detected by clinical symptoms or biochemical methods. In tyrosinemia reversion of the primary defect to a normal genotype with functional FAH enzyme would give this cell the necessary selective advantage. Whereas the reversion of the mutation in one cell does not eliminate the risk of cancer development in non-reverted cells, the existence of a normally functioning cell pool, may prevent liver failure and thereby influence the course of the disorder. If the reversion of the genetic defect in tyrosinemia results mainly from accelerated cell regeneration such an event may not be exclusive to tyrosinemia and should be sought for in other genetic diseases in tissues with an induced, or natural high, rate of cell replication. The reversion of the disease causing point mutations in tyrosinemia, with the potential of self-induced cure, may be the counterpart to the carcinogenesis of the disorder, possibly reflecting the same process.

\section{Acknowledgments}

We would like to thank Drs. S. G. Hubscher (University of Birmingham, Birmingham, UK), G. Gray (Children's Hospital, Birmingham, UK),
Dr. B. Wilcken (Oliver Latham Laboratory, Sydney, Australia), and Dr. G. Thompson (The Murdoch Institute, Royal Children's Hospital, Melbourne, Australia) for liver tissue blocks from their tyrosinemia patients.

\section{References}

1. Goldsmith, L. A., and C. Laberge. 1989. Tyrosinemia and related disorders. In The Metabolic Basis of Inherited Disease. C. R. Scriver, A. L. Beaudet, W.-S. Sly, and D. Valle, editors. McGraw-Hill Inc., New York 547-562.

2. Kvittingen, E. A., H. Rootwelt, P. Brandtzaeg, A. Bergan, and R. Berger. 1993. Hereditary tyrosinemia type I. Self-induced correction of the fumarylacetoacetase defect. J. Clin. Invest. 91:1816-1821.

3. Rootwelt, H., T. Kristensen, R. Berger, K. Høie, and E. A. Kvittingen. Tyrosinemia type I-complex splicing defects and a missense mutation in the fumarylacetoacetase gene. Hum. Genet. 94:235-239.

4. Grompe, M., and M. Al-Dhalimy. 1993. Mutations of the fumarylacetoacetate hydrolase gene in four patients with tyrosinemia type I. Hum. Mut. 2:85-93.

5. Rootwelt, H., R. Berger, G. Gray, D. A. Kelly, T. Coskun, and E. A Kvittingen. Novel splice, missense and nonsense mutations in the fumarylacetoacetase gene causing tyrosinemia type I. Am. J. Hum. Genet. In press.

6. Seeberg, E., and R. P. P. Fochs. 1990. Acetylaminofluorene bound to different guanines of the sequence GGCGCC is excised with different efficiencies by the UvrABC excision nuclease in a pattern not correlated to potency of mutation induction. Proc. Natl. Acad. Sci. USA. 87:191-194.

7. Fochs, R. P. P. 1984. DNA binding spectrum of the carcinogen $\mathrm{N}$-acetoxy$\mathrm{N}$-2-acetylaminofluorene significantly differs from the mutation spectrum. J. Mol Biol. 177:173-180.

8. Macvicar, D., C. Dicks-Mireaux, J. V. Leonard, and D. G. D. Wight. 1990. Hepatic imaging with computed tomography of chronic tyrosinaemia type I. Br. J. Radiol. 63:605-608. 